\title{
Laplace Problems for Regular Lattices with an Even Number of Different Obstacles
}

By

\author{
G. Caristi and M. Stoka \\ (vorgelegt in der Sitzung der math.-nat. Klasse am 17. Juni 2010 durch \\ das w. M. August Florian)
}

\begin{abstract}
In this paper we consider some regular lattices with fundamental cell with a even number of obstacles. In particular we obtain the Laplace probability.

Key words: Geometric probability, stochastic geometry, random sets, random convex sets and integral geometry.
\end{abstract}

\section{Section}

Let $\Re_{1}(a, b, c)$ be the regular lattice with fundamental cell is as in Fig. 1.

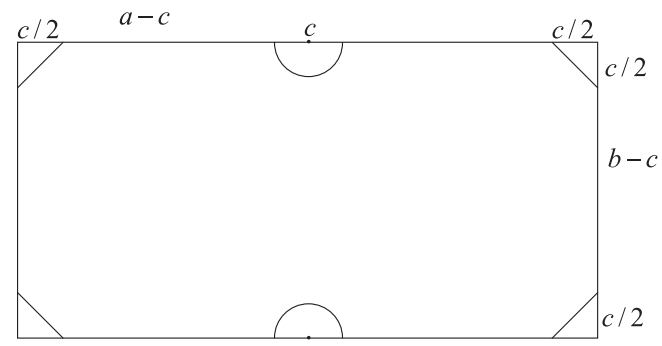

Fig. 1 
Denoting with $C_{0}^{(1)}$ the fundamental cell of this lattice, we have:

$$
\text { area } C_{0}^{(1)}=2 a b-\frac{(2+\pi) c^{2}}{4} .
$$

The cell $C_{0}^{(1)}$ has six obstacles that are quarter-squares with diagonal of length $c$ with $c<\min (a, b)$ and semi circles with diameter $c$.

Considering a segment $s$ of random position and of constant length $l$ with $c<l<\min (a, b)$, we want compute the probability that this segment intersects a side of lattice; obviously this probability is equal to probability $P_{\text {int }}^{(1)}$ that the segment $s$ intersects the bounderay of the fundamental cell.

The position of the segment $s$ is determinated by the middle point $O$ and by the angle $\varphi$ that the segment forms with the axis $x$. We consider the limit positions of the segment $s$ that corrisponde at angle $\varphi$ and let $\widehat{C}_{0}^{(1)}(\varphi)$ the determinated figure from these positions (Fig. 2):

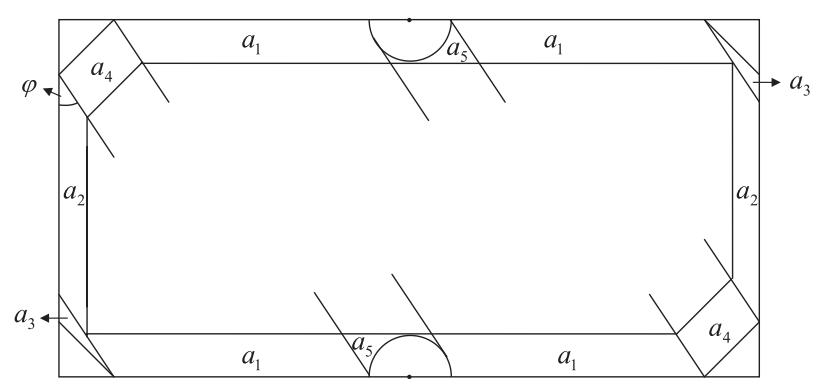

Fig. 2

From this figure we can write:

$$
\begin{aligned}
\operatorname{area} \widehat{C}_{0}^{(1)}(\varphi)= & \operatorname{area} C_{0}^{(1)} \\
& -\left[4 \operatorname{area} a_{1}(\varphi)+2 \operatorname{area} a_{2}(\varphi)+2 \operatorname{area} a_{3}(\varphi)\right. \\
& \left.+2 \operatorname{area} a_{4}(\varphi)+2 \operatorname{area} a_{5}(\varphi)\right] .
\end{aligned}
$$

Considering some results that we have obtained in a previous paper [1], follow that:

$$
\begin{gathered}
\operatorname{area} a_{1}(\varphi)=\frac{(a-c) l}{2} \cos \varphi, \quad \operatorname{area}\left[a_{2}(\varphi)+a_{3}(\varphi)\right]=\frac{(b-c) l}{2} \sin \varphi \\
\operatorname{area} a_{4}(\varphi)=\frac{c l}{4}(\sin \varphi+\cos \varphi)
\end{gathered}
$$


But from Fig. 3:

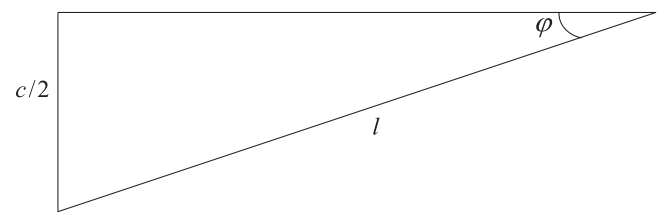

Fig. 3

we have $c=2 l \sin \varphi$, hence

$$
\operatorname{area} a_{4}(\varphi)=\frac{c l}{4}(\sin \varphi+2 \cos \varphi)-\frac{l^{2}}{4} \sin 2 \varphi
$$

In order to compute area $a_{5}(\varphi)$, we consider the figure

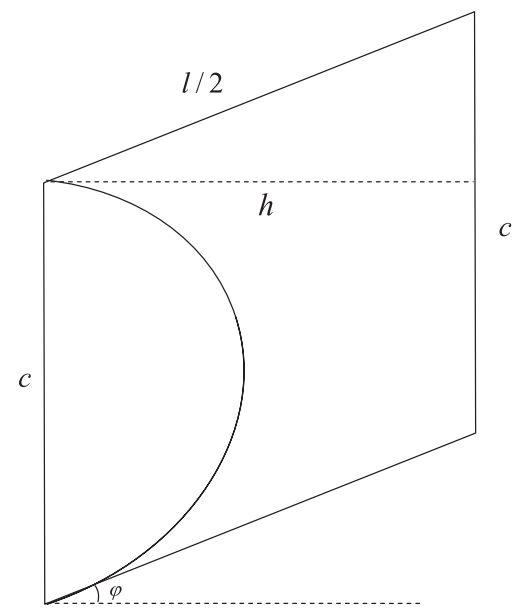

Fig. 4

From here follows $h=\frac{l}{2} \cos \varphi$, hence

$$
\text { area } a_{5}(\varphi)=\frac{c l}{2} \cos \varphi-\frac{\pi c^{2}}{8}
$$

Replacing in the formula (1) le expressions (2), (3) and (4) we obtain

$$
\begin{aligned}
\operatorname{area} \widehat{C}_{0}^{(1)}(\varphi)= & \operatorname{area} C_{0}^{(1)} \\
& -\left[2 a l \cos \varphi+\left(b-\frac{c}{2}\right) l \sin \varphi-\frac{l^{2}}{2} \sin 2 \varphi-\frac{\pi c^{2}}{4}\right] .
\end{aligned}
$$


Denoting with $M_{1}$ the set of segments $s$ whose the middle point are in $C_{0}^{(1)}$ and $N_{1}$ the set of segments $s$ completely contained in $C_{0}^{(1)}$, we have that:

$$
P_{\text {int }}^{(1)}=1-\frac{\mu\left(N_{1}\right)}{\mu\left(M_{1}\right)},
$$

where $\mu$ is the Lebesgue measure in Euclidean plane [3].

In order to compute the measures $\mu\left(M_{1}\right)$ and $\mu\left(N_{1}\right)$ we use the Poincaré kinematic measure [2]

$$
d K=d x \wedge d y \wedge d \varphi
$$

where $x, y$ are the coordinates of $O$ and $\varphi$ the defined angle.

Since $\varphi \in\left[0, \frac{\pi}{2}\right]$, we have:

$$
\mu\left(M_{1}\right)=\int_{0}^{\frac{\pi}{2}} d \varphi \iint_{\left\{(x, y) \in C_{0}^{(1)}\right\}} d x d y=\frac{\pi}{2} \operatorname{area} C_{0}^{(1)}=\frac{\pi}{2}\left[2 a b-\frac{(2+\pi) c^{2}}{4}\right] .
$$

and, considering the (5)

$$
\begin{aligned}
& \mu\left(N_{1}\right)=\int_{0}^{\frac{\pi}{2}} d \varphi \iint_{\left\{(x, y) \in \hat{C}_{0}^{(1)}(\varphi)\right\}} d x d y=\int_{0}^{\frac{\pi}{2}} \operatorname{area} \widehat{C}_{0}^{(1)}(\varphi) d \varphi \\
& =\frac{\pi}{2}\left[2 a b-\frac{(2+\pi) c^{2}}{4}\right]-\int_{0}^{\frac{\pi}{2}}\left[2 a l \cos \varphi+\left(b-\frac{c}{2}\right) l \sin \varphi-\frac{l^{2}}{2} \sin 2 \varphi-\frac{\pi c^{2}}{4}\right] d \varphi \\
& =\frac{\pi}{2}\left[2 a b-\frac{(2+\pi) c^{2}}{4}\right]-\left[2 a l \sin \varphi-\left(b-\frac{c}{2}\right) l \cos \varphi+\frac{l^{2}}{4} \cos 2 \varphi-\frac{\pi c^{2}}{4} \varphi\right] \\
& =\frac{\pi}{2}\left[2 a b-\frac{(2+\pi) c^{2}}{4}\right]-\left[\left(2 a+b-\frac{c}{2}\right) l-\frac{l^{2}}{2}-\frac{\pi^{2} c^{2}}{8}\right] .
\end{aligned}
$$

The formulas (6), (7) and (8) give us that:

$$
P_{\text {int }}^{(1)}=\frac{2\left(2 a+b-\frac{c}{2}\right) l-l^{2}-\frac{\pi^{2} c^{2}}{4}}{\pi\left[2 a b-\frac{(2+\pi) c^{2}}{4}\right]} .
$$

When $c \rightarrow 0$, the obstacles becames points and the fundamental cell becames a rectangle with side $2 a$ and $b$. In this case the probability (9) becomes the Laplace probability:

$$
P=\frac{2(2 a+b) l-l^{2}}{2 \pi a b} .
$$




\section{Section}

Let $\Re_{2}(a, b, c, n)$ be the regular lattice with fundamental cell $C_{0}^{(2)}$ a rectangle with side $(n+1) a$ e $b$ and with $4(n+1)$ obstacles: $2 n$ semi circles with the radius $\frac{c}{2}, 2$ quarter-circles with the same radius, $2 n$ semi square and 2 quarter-square with the diagonal $c$ with $c<\min [(n+1) a, b]$ (Fig. 5):

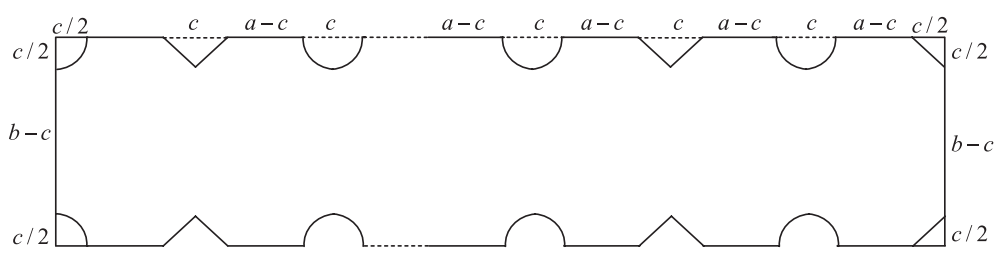

Fig. 5

We have:

$$
\operatorname{area} C_{0}^{(2)}=(n+1) a b-\frac{c^{2}}{4}\left(n+1-\frac{4 n+1}{4} \pi\right) .
$$

In the same way of the Section 1, considering a segment $s$ of random position and of constant length $l$ with $c<l<\min [(n+1) a, b]$.

We want compute the probability that this segment intersects a side of lattice, obviously this probability is equal to probability $P_{\text {int }}^{(2)}$ that the segment $s$ intersects the bounderay of the fundamental cell.

The position of the segment $s$ is determinated by the middle point $O$ and by the angle $\varphi$ that the segment forms with the axis $x$. We consider the limit positions of the segment $s$ that corrisponde at angle $\varphi$ and let $\widehat{C}_{0}^{(2)}(\varphi)$ the determinated figure from these positions (Fig. 6):

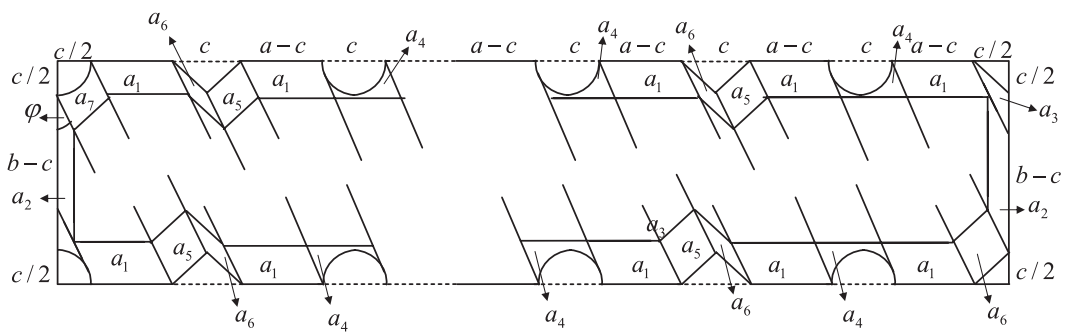

Fig. 6 
From this figure we can write:

$$
\begin{aligned}
\operatorname{area} \widehat{C}_{0}^{(2)}(\varphi)= & \operatorname{area} C_{0}^{(2)}-2(n+1) \operatorname{area} a_{1}(\varphi)+2 \operatorname{area} a_{2}(\varphi) \\
& +\operatorname{area} a_{3}(\varphi)+2 \text { narea } a_{4}(\varphi)+n \operatorname{area} a_{5}(\varphi)+n \operatorname{narea} a_{6}(\varphi) \\
& +\operatorname{area} a_{7}(\varphi)+\operatorname{area} a_{8}(\varphi)+\operatorname{area} a_{9}(\varphi) .
\end{aligned}
$$

Considering of some results that we have obtained in a previous paper [1], we have:

$$
\begin{gathered}
\operatorname{area} a_{1}(\varphi)=\frac{(a-c) l}{2} \cos \varphi, \quad \operatorname{area} a_{2}(\varphi)=\left(b-\frac{c}{2}-l \cos \varphi\right) \frac{l}{2} \sin \varphi \\
\operatorname{area} a_{3}(\varphi)=\frac{c l}{2} \cos \varphi-\frac{c^{2}}{8}
\end{gathered}
$$

and

$$
\begin{aligned}
& \operatorname{area} a_{7}(\varphi)=\frac{c l}{4}(\sin \varphi+\cos \varphi)-\frac{\pi c^{2}}{8}+\frac{c^{2}}{8}, \\
& \operatorname{area} a_{8}(\varphi)=\frac{c l}{4} \cos \varphi-\frac{\pi c^{2}}{16}, \quad \operatorname{area} a_{9}(\varphi)=\frac{c l}{4}(\sin \varphi+\cos \varphi) .
\end{aligned}
$$

In order to compute area $a_{4}(\varphi)$ we consider Fig. 7:

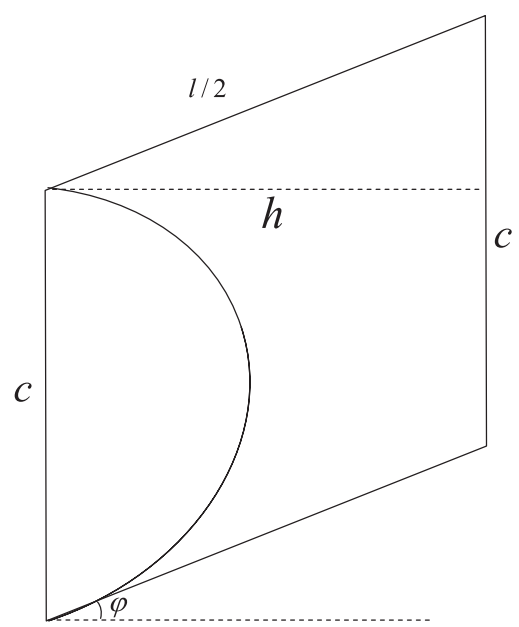

Fig. 7 
From here follows $h=\frac{l}{2} \cos \varphi$, hence

From Fig. 8:

$$
\text { area } a_{4}(\varphi)=\frac{c l}{2} \cos \varphi-\frac{\pi c^{2}}{8}
$$

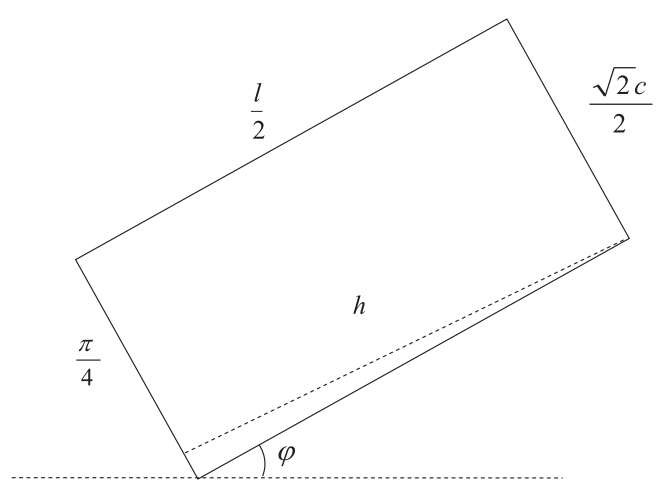

Fig. 8

we have

$$
h=\frac{l}{2} \sin \left(\frac{\pi}{4}+\varphi\right)=\frac{l \sqrt{2}}{4}(\sin \varphi+\cos \varphi)
$$

then

$$
\operatorname{area} a_{5}(\varphi)=\frac{c l}{4}(\sin \varphi+\cos \varphi)
$$

In the end, from Fig. 9:

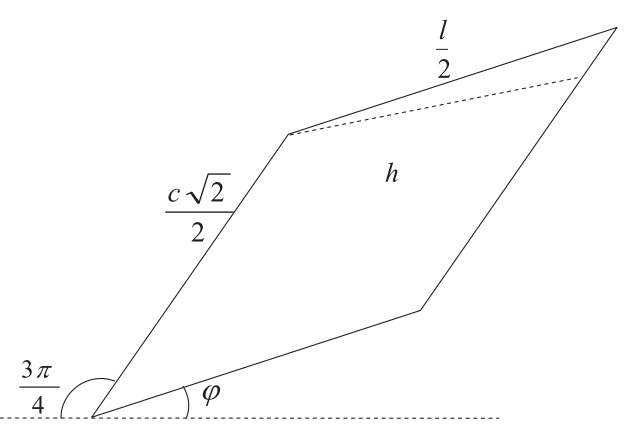

Fig. 9 
follows:

$$
h=\frac{l}{2} \sin \left(\frac{\pi}{4}-\varphi\right)=\frac{l \sqrt{2}}{4}(\cos \varphi-\sin \varphi)
$$

then

$$
\operatorname{area} a_{6}(\varphi)=\frac{c l}{4}(\cos \varphi-\sin \varphi)
$$

Replacing in the (10) the expression (11), (12), (13) and (14) we obtain:

$$
\begin{gathered}
\operatorname{area} \hat{C}_{0}^{(2)}(\varphi)=\operatorname{area} C_{0}^{(2)}-\left\{\left[(n+1) a+\frac{2 n+1}{4} c\right] l \cos \varphi+b l \sin \varphi\right. \\
\left.-\frac{l^{2}}{2} \sin 2 \varphi-\frac{(4 n+3) \pi c^{2}}{16}\right\}
\end{gathered}
$$

Denoting with $M_{2}$ the set of segments $s$ whose the middle point are in $C_{0}^{(2)}$ and $N_{2}$ the set of segments $s$ completely contained in $C_{0}^{(2)}$, we have, as above, that:

$$
P_{\text {int }}^{(2)}=1-\frac{\mu\left(N_{2}\right)}{\mu\left(M_{2}\right)}
$$

Since $\varphi \in\left[0, \frac{\pi}{2}\right]$, we have:

$$
\begin{aligned}
\mu\left(M_{2}\right) & =\int_{0}^{\frac{\pi}{2}} d \varphi \iint_{\left\{(x, y) \in C_{0}^{(2)}\right\}} d x d y=\frac{\pi}{2} \operatorname{area} C_{0}^{(2)} \\
& =\frac{\pi}{2}\left[(n+1) a b-\frac{c^{2}}{4}\left(n+1-\frac{4 n+1}{4} \pi\right)\right]
\end{aligned}
$$

and considering the (15), 


$$
\begin{aligned}
\mu\left(N_{2}\right)= & \int_{0}^{\frac{\pi}{2}} d \varphi \iint_{\left\{(x, y) \in \hat{C}_{0}^{(2)}(\varphi)\right\}} d x d y=\int_{0}^{\frac{\pi}{2}} \operatorname{area} C_{0}^{(2)}(\varphi) d \varphi \\
= & \frac{\pi}{2} \operatorname{area} C_{0}^{(2)}-\int_{0}^{\frac{\pi}{2}}\left\{\left[(n+1) a+\frac{2 n+1}{4} c\right] l \cos \varphi+b l \sin \varphi\right. \\
= & \left.-l^{2} \sin 2 \varphi-\frac{(4 n+3) \pi c^{2}}{16}\right\} d \varphi \\
& \operatorname{area} C_{0}^{(2)}-\left\{\left[(n+1) a+b+\frac{2 n+1}{4} c\right] l-\frac{l^{2}}{2}-\frac{(4 n+3) \pi^{2} c^{2}}{32}\right\} .
\end{aligned}
$$

The formulas (16), (17) and (18) give us that:

$$
P_{\mathrm{int}}^{(2)}=\frac{2\left[(n+1) a+b+\frac{2 n+1}{4} c\right] l-l^{2}-\frac{(4 n+3) \pi^{2} c^{2}}{16}}{\pi\left[(n+1) a b-\frac{c^{2}}{4}\left(n+1-\frac{4 n+1}{4} \pi\right)\right]} .
$$

When $c \rightarrow 0$, the obstacles becames points and the fundamental cell becames a rectangle with side $(n+1) a$ and $b$. In this case the probability (18) becomes the Laplace probability:

$$
P=\frac{2[(n+1) a+b] l-l^{2}}{(n+1) \pi a b} .
$$

\section{References}

[1] CARISTi G, StoKA M. A Laplace type problem for a regular lattice with obstacles (I), Atti. Acc. Sci. Torino (to appear)

[2] Poincaré H. (1912) Calcul des probabilitiés, ed. 2, Carré, Paris

[3] STOKA M. (1975-1976) Probabilités géométriques de type Buffon dans le plan euclidien, Atti. Acc. Sci. Torino, T. 110, pp. 53-59

Author's addresses: G. Caristi, Department SEA, University of Messina, Via dei Verdi n. 75, 98122 Messina, Italy. E-Mail: gcaristi@unime.it; M. Stoka, Accademia delle Scienze di Torino, Via Maria Vittoria 3, 10123 Torino, Italy. 